\title{
DERMATOLOGICAL SIGNS OF THE MOST FREQUENTLY SYSTEMIC BACTERIAL INFECTIONS
}

\author{
Sonya Marina ${ }^{1}$, Vessela Ouzounova-Raykova ${ }^{1,2}$ \\ ${ }^{1}$ Department of Dermatology and Venerology, Alexandrovska University Hospital, \\ Medical University of Sofia, ${ }^{2}$ Department of Medical Microbiology, Medical University \\ of Sofia
}

\begin{abstract}
The dermatologic complaints are a common reason for consultation with the general practitioner or dermatologist. The aim of this article is to highlight the more common, classic and important systemic bacterial infections presenting with dermatology manifestations. The finding of the specific skin signs may aid in the elucidation and the evaluation of underlying disease and the prompt and adequate start of patients therapy.
\end{abstract}

Keywords: systemic bacterial infections, dermatological signs

\section{INTRODUCTION}

The skin provides a window into the patient and helps in the diagnosis of diseases of all organ systems including the systemic bacterial ones. Skin manifestations of systemic bacterial diseases are varied, specific and nonspecific. Similar clinical manifestations may be caused by different bacteria, and the same bacteria may be responsible for different manifestations. Therefore, the skin signs of the systemic bacterial infections may be classified according to the bacteria or the clinical syndrome.

\section{Bacterial endocarditis}

Bacterial endocarditis is an infection of the lining of the heart. The bacteria gain access to the heart via

\author{
Address for correspondence: \\ Vessela Ouzounova-Raykova \\ Department of Dermatology and Venerology \\ Medical University of Sofia, Bulgaria \\ 2 Zdrave Str. \\ 1431, Sofia, Bulgaria \\ e-mail:pumpi@abv.bg \\ phone: 0035929172580
}

Received: January 7, 2014

Accepted: February 11, 2014 the bloodstream. An infection elsewhere in the body may or may not be apparent. While some bacteria can cause infection in normal heart valves, bacterial endocarditis more commonly affects patients with abnormal valves as a result of previous damage, septal defects, chordae tendinae, and mural endocardium. The responsible bacteria include various species - Staphylococcus, Streptococcus, Enterococcus, Pseudomonas, Bartonella, Coxiella, Chlamydia, Legionella, Brucella, HACEK group, etc. (2).

The symptoms include fever, lethargy, shortness of breath, chest pain, palpitations, heart murmur, splenomegaly. Early diagnosis, before the appearance of complications, is the key to proper management. The appearance of painful, red-purple, slightly raised, tender pea-sized nodules known as Osler's nodes distributed over the pads of the fingers and toes, the tenar and hypotenar eminences and along the sides of the fingers are helpful for the diagnosis (13). Pain often precedes the development of the visible lesion by up to 24 hours. They can occur at any time during the course of endocarditis and last from hours to several days.

In contrast the Janeway lesions (13) are nontender, often haemorrhagic and occur mostly on the palms and soles. They tend to last days to weeks 
before healing completely. They are more commonly seen in acute endocarditis.

Conglomeration of petechiae in the skin or mucous membranes and also in the conjunctivae occur in as high as $80 \%$ of the cases. The socalled Splinter haemorrhages which are fine linear haemorrhagic lesions under the finger nails and toenails may also be present (17).

\section{Meningococcal infection}

Meningococcal disease is caused by virulent bacteria commonly found in the back of the throat - N. miningitidis. Anyone at any time is at risk of contracting meningococcal disease. There still is no understanding as to why it is possible to live with these bacteria in the airways meanwhile in rare cases the natural defence system is unable to cope with them.

Meningococcal disease commonly takes two forms: septicaemia and meningitis. The most striking sign on physical examination, no matter the form, is the skin eruption (5). This is present in the majority of patients with only a few patients described without it and develops thanks to the endotoxin released by the microorganism on its death or disintegration. The eruption may take a variety of forms, but the most common are petechial and purpuric lesions. Pinkyellowish macules, wheals or nodules that resemble erythema nodosum may occur before petechiae and ecchymoses. Rarely, vesicular, pustular or bullous lesions may appear. The specific perechiae might be found anywhere on the skin or mucous membrane, but predominate on wrists and ankles.

\section{Disseminated gonococcal infection}

Disseminated infection is rare (1-3\% of persons who have gonorrhea). Septic emboli can cause polyarticular tenosynovitis and dermatitis. Symptoms of disseminated infection can range from slight joint pain, a few skin lesions, and no fever to overt polyarthritis and high fever (1). Patients with disseminated gonorrhea usually have no urogenital symptoms.

The skin lesions typically are few and are limited to the extremities. They start as papules and progress into hemorrhagic pustules. Bullae, petechiae or necrotic lesions also may appear. The skin lesions usually are resolved if the gonorrhea continues to disseminate. Skin lesions and blood cultures usually are negative for $\mathrm{N}$. gonorrhoeae. Disseminated gonorrhea also may present as bacterial endocarditis, meningitis and myocarditis.

\section{Dermal abscesses}

These are rarely associated with disseminated bacterial infection as a secondary localisation. They may appear as unique or as multiple. According to the isolated bacteria and the frequency of the clinical manifestation three types of abscesses may be distinguished.

Acute abscesses form the inflammatory dermal mass during the patients bacteriemia. These abscesses are rare. They could be caused by any pyogenic bacteria but most frequently by $S$. Aureus (15).

Sub-acute abscesses occur several days to weeks after the bacteriemia and are caused mainly by Nocardia spp. or non-tuberculosis Mycobacteria (18). If there is an immunosupression in the patient the sub-acute abscesses may be caused by Mycobacteria.

The so called cold abscesses (14) occur months after the systemic dissemination of the microorganism and mainly are caused by M. tuberculosis (11).

\section{Ecthyma gangrenosum}

This is a cutaneous infection commonly associated with Pseudomonas bacteraemia, but S. aureus, S. pyogenes, A. hydrophila, C. violaceum, A. fumigatus, C. albicans, Mucor and Rhizopus spp., HSV were documented too (3).

Ecthyma gangrenosum usually occurs in patients with profound and prolonged neutropenia. Impaired immunity leads to increased susceptibility to infections. Breakdown of mechanical defensive barriers such as skin and mucosa allows entry of infectious organisms. The lesions result from bacterial invasion of the walls of arteries and veins in the skin and subcutaneous tissue. The bacterial invasion may come from inside the vessel in the case of septicaemia, or by direct inoculation of bacteria through the skin.

The characteristic lesions of ecthyma gangrenosum are haemorrhagic pustules that evolve into necrotic ulcers (14). The initial lesions appear 
as painless, round, red patches in the skin which rapidly become pustular with surrounding redness. A haemorrhagic focus appears in the centre, forming a blister. As the haemorrhagic blister spreads peripherally, it evolves into a gangrenous ulcer with a black scab surrounded by a red halo. An early lesion may transform into a necrotic ulcer in as little as 12 hours. Ecthyma gangrenosum may appear at any site but mainly affects the anogenital area and armpits.

\section{Disseminated miliary tuberculosis of the skin}

This is an uncommon form of tuberculosis characterized by a papulopustular eruption haematogenous dissemination of $\mathrm{M}$. tuberculosis to multiple organs and often a fatal outcome. This clinical entity, which occurred mainly in infants in the prechemotherapy era, has reemerged among HIVinfected patients in recent years. In miliary tuberculosis lungs are affected but meninges, liver and spleen can be involved. Two factors are responsible for the appearance of this tuberculosis form: severe immune dysfunction and infection with resistant to antituberculous drugs M. tuberculosis (10). The typical eruptions consist of discrete papulo-pustules on erythematous bases. Other manifestations include macules, pustular lesions, ulcerations, purpuric lesions and sub-cutaneous nodules. The lesions are distributed over the trunk and extremities, but any part of the body can be affected and usually do not exceed 20-30 in number.

\section{Toxic shock syndrome}

TSS is a sudden, potentially fatal condition due to exotoxins produced by S. aureus (9). Not all strains cause TSS. In fact, S. aureus is normally and harmlessly present in the vagina. But the producing TSS-exotoxin bacteria are not rare.

TSS is still primarily a disease of menstruating women using tampons, but also menstrual sponges, diaphragms and cervical caps. A woman who has recently given birth also is at risk for TSS. The condition is not limited to these factors alone men and women exposed to $S$. aureus bacteria while recovering from surgery, burn, open wound or the use of a prosthetic devices may develop this condition.
Because TSS gets worse quickly and can be deadly within 2 days it is good to know the symptoms - sudden fever over $39^{\circ} \mathrm{C}$, hypotension, macular erythema that peels after 1-2 weeks and multiorgan dysfunction (19). The skin manifestations include generalised erythema that can progress to desquamation with involvement of palms and soles. It can be found in several areas of the body or just in the armpits or the groin. Transitient alopecia, fallingof the nails and an increased sweating of hands and feet have been described too.

\section{Streptococcal Toxic Shock-like Syndrome}

TSS is caused by S. aureus. Toxic shock-like syndrome (TSLS) has the same clinical manifestation as TSS but is caused by Streptococcus (predominantly Group A, C, G) that are toxin-mediated, associated with superantigens (19). In the past few years different streptococcal mitogens were established as stimulation inducers of large $\mathrm{T}$ cells populations which produce toxic concentrations of cytokines that cause the TSLS manifestation.

\section{Staphylococcal scalded skin syndrome}

SSSS encompasses a spectrum of superficial blistering skin disorders caused by the exfoliative toxins of some $S$. aureus strains (4). This syndrome of acute exfoliation typically follows the erythematous cellulitis. Severity of SSSS varies from a few blisters localised to the site of infection to a severe exfoliation affecting almost the entire body.

Two exfoliative toxins have been isolated but the exact mechanism of the exfoliation, until recently had been uncertain. The toxins likely act as proteases that target the desmoglein-1, found only in the superficial epidermis. The relative quantity of desmoglein-1 in the skin differs with age and may partially explain the increased frequency of SSSS in children younger than 5 years. Lifelong protective antibodies against staphylococcal exotoxins are usually acquired during childhood which makes SSSS much less common in adults. It is theorised that immature renal function in this age may contribute to impaired clearance of circulating exotoxins, contributing to more extensive disease. Another theory suggests that the exfoliative toxins may possess a superantigenic activity. So, 
immunocompromised individuals and individuals with renal failure, regardless of age, may also develop SSSS.

According to the clinical manifestation SSSS usually starts with fever, irritability and widespread redness of the skin. Within 24-48 hours bullae are formed. These easily rupture leaving an area that looks like a burn. Characteristics of the rash include tissue paper-like wrinkling of the skin followed by the appearance of large bullae in the armpits, groin and body orifices such as the nose and ears. Rash spreads to other parts of the body including the arms, legs and trunk. In newborns, lesions are often found in the diaper area or around the umbilical cord. Top layer of skin begins peeling off in sheets, leaving exposed a moist, red and tender area.

\section{Scarlet fever}

This contagious infection occurs mostly in children and is caused by S. pyogenes (12). It is spread by contact with nasal or mouth fluids from an infected person. Symptoms typically appear three days after exposure to an ill person, although this incubation period can be from one to seven days. Illness usually begins with a fever and sore throat, chills, vomiting and abdominal pain. The tongue may have a whitish coating, a reason for the "strawberry"like appearance. The throat and tonsils may be red, sore and painful.

One or two days after the illness begins, characteristic red rash appears (although the rash can appear before the illness or up to 7 days later). The rash may first appear on the neck, under arm or groin, then spreads over the body. Typically, the rash begins as small, flat red blotches which gradually become fine bumps. Although the cheeks might have a flushed appearance, there may be a pale area around the mouth. Underarm, elbow and groin skin creases may become brighter red than the rest of the rash. The scarlet fever rash generally fades in about 7 days and the skin may peel around the finger tips, toes, and groin area.

\section{Purpura fulminans}

Purpura fulminans is a rare rapidly progressive syndrome of intravascular thrombosis and hemorrhagic infarction of the skin. It is accompanied by vascular collapse and disseminated intravascular coagulation. It usually occurs in children, but has also been noted in adults. The 3 forms of this disease are classified by the triggering mechanisms.

Neonatal purpura fulminans is associated with a hereditary deficiency of the natural anticoagulants protein $\mathrm{C}$ and $\mathrm{S}$, as well as antithrombin III. The second type is idiopathic or chronic purpura fulminans, which follows a bacterial/viral illness and occurs after a latent period. It usually follows an initiating febrile illness that manifests with rapidly progressive purpura, which may lead to skin necrosis, gangrene of limbs or digits and major organ dysfunction. The most common form of purpura fulminans occurs superimposed on a bacterial infection and has been called acute infectious purpura fulminans. In this illness, the balance of anticoagulant and procoagulant endothelial cell activity is disturbed. This disturbance is precipitated by bacterial endotoxin and mediated by various factors that include the inflammatory cytokines IL12 , interferon- $\gamma$, TNF- $\alpha$ and IL-1 which consume ATIII as well as proteins $\mathrm{C}$ and S. Microemboli and direct bacterial damage to vessels have also been linked with this process. The two most common causes of acute infectious purpura fulminans are meningococcus and varicella. Gram-negative bacilli, Staphylococci, Rickettsia species, Sreptococci and measles have also been found as causative agents.

Over time, the term purpura fulminans has been applied to cases of purpura fulminans that occurs in the face of overwhelming sepsis (6). The 4 primary features of this syndrome are large purpuric skin lesions, fever, hypotension and disseminated intravascular coagulation. Meningococcemia is generally more predisposed than other bacteria to cause dysfunction of the activated protein $\mathrm{C}$ pathway. More recently S. aureus has been associated with purpura fulminans with accompanying TSS.

\section{Secondary syphilis}

Secondary syphilis is typically a systemic disease presenting with a variety of symptoms - malaise, sore throat, headache, weight loss, low-grade fever, 
pruritus and muscle aches, lymph node enlargement in addition to the dermatologic manifestations.

Lesions result from the haematogenous dissemination of treponemas from syphilitic chancres. Secondary-stage lesions generally appear 4-10 weeks after the initial start of primary lesions. However, in some patients with disseminated lesions there is an overlap and a careful examination may disclose primary chancre. Malignant syphilis, wherein disseminated lesions resemble primary chancres, is rare, despite several recent case reports in the literature. The tendency for the skin to be the most extensively involved of all organs may reflect the slightly lower temperature.

The initial finding in disseminated syphilis is an evanescent macular rash that is often overlooked (8). The macular lesions of early secondary syphilis differ from all other secondary lesions in several aspects. Were it not for the fact that macular lesions are often overlooked or misdiagnosed, there is little question that they are probably the most common of all secondary lesions. A few days later, a symmetric papular eruption appears, involving the entire trunk and the extremities, including the palms of the hands and the soles of the feet. They are generally scaly, although they may be smooth, follicular, or rarely, pustular. Vesicles usually do not occur, although vesiculopustular lesions are seen on rare occasions and are common on the palms or soles. Mucosal lesions are also quite common and characteristic of secondary syphilis (7). Alopecia also occurs in untreated cases, reflecting involvement of hair follicles.

Condyloma latum refers to one or more large, raised, white or gray lesions found in warm, moist areas. These lesions, originally described as a manifestation of well-established secondary syphilis, reflect a local breakdown of secondary lesions with extension of infection in areas of tissue trauma, most frequently involving the axilla and groin. More common, at present, are condylomatous lesions that appear in the perineum or around the anus before or soon after the generalised lesions.

\section{Rickettsial diseases}

These encompass a group of diseases caused by Rickettsiae. Rickettsial diseases vary considerably in severity from self-limiting mild illnesses to severe life-threatening infections.

Vasculitis is the basic pathogenetic mechanism. Vasculitis is responsible for skin rash, microvascular leakage, edema, tissue hypoperfusion and endorgan ischemic injury. Formation of thrombi can lead to tissue infarction and hemorrhagic necrosis. Inflammation and vascularleakageleads to interstitial pneumonitis, noncardiogenic pulmonary edema, cerebral edema and meningoencephalitis. Infection of endothelial cells also induces procoagulant activity that promotes coagulation factor consumption, platelet adhesion and leucocyte emigration and may result in clinical syndrome similar to disseminated intravascular coagulation.

Early signs and symptoms of these infections are nonspecific and mimic benign viral illnesses, making diagnosis difficult. Fever, headache, myalgia and rash are considered as hallmark of rickettsial disease, but the rash is neither seen at presentation nor in all the patients. Thus it should be remembered that spotted fevers could be spotless too. Rash usually becomes apparent after 3-5 days of onset of symptoms. Initially rash is in the form of pink, blanching, discrete macules which subsequently becomes maculopapular, petechial or hemorrhagic. Sometimes palpable purpura typical of vasculitis is seen (16). Occasionally petechiae enlarge to ecchymosis and gangrenous patches may develop. Rarely gangrene of digits, earlobes, scrotum, nose or limbs may occur secondary to vasculitis and thrombosis. Distribution of rash is initially near ankles, lower legs and wrists. Thereafter rash spreads centripetally to involve whole body. Presence of rash on palms and soles, considered so typical of rickettsial disease, can be seen in other diseases like infective endocarditis, syphilis, meningococcemia, enteroviral diseases and adverse drug reactions. The rash of typhus group rickettsioses is quite atypical, initially appearing on the trunk, spreading centrifugally and usually sparing palms and soles. A necrotic eschar at the inoculating site is seen in variable proportion of Indian tick typhus, scrub typhus and rickettsialpox cases. The site of initial tick bite is inapparent in 
other rickettsial infections. Eschar, a black necrotic area, resembles the skin burn of cigarette butt. A necrotic eschar usually has an erythematous rim and is associated with regional lymphadenopathy.

\section{CONCLUSIONS}

It was beyond the scope of this review to cover all potential skin manifestations of systemic bacterial disease. This article highlights the more common, classic and important manifestations of the systemic bacterial infections. It is important not to overlook dermatological problems. They could be timely clues to discovering serious systemic problems because: systemic infections often present with cutaneous signs; the careful identification of diagnostic skin clues is important for the diagnosis of infections; the dissemination of the cutaneous infections may demonstrate the immune status of patients; and the timely diagnosis may help in the treatment of the patient and prevention of more severe complications.

\section{REFERENCES}

1. Bleich, A.T., J.S. Sheffield, G.D. Jr. Wendel, A. Sigman, F.G. Cunningham. Disseminated gonococcal infection in women. - Obstet Gynecol, 2012;119(3):597-602.

2. Brouqui, P., D. Raoult. Endocarditis due to rare and fastidious bacteria. - Clin. Microbiol Rev, 2001;14(1):177-207.

3. Bucak, I.H., G. Tümgör, E. Mengen, F. Temiz, M. Turgut. Ecthyma gangrenosum in a previously healthy pediatric patient and associated facial paralysis and persistent hyperplastic primary vitreous. - Am J Case Rep, 2012;13:250-253.

4. Courjon, J., T. Hubiche, A. Phan et al. Skin findings of Staphylococcus aureus toxin-mediated infection in relation to toxin encoding genes. Pediatr Infect Dis J, 2013;32(7):727-30.

5. Drage, L.A., J.B. Bundrick, S.C. Litin . Clinical pearls in dermatology. - Mayo Clin Proc, 2012;87(7):695-9.

6. Fitzgerald, C.J., T.V. Pranikoff, G.A. Ross, S. Mou, L.B. Givner, A.K. Shetty. Purpura fulminans caused by community-associated methicillinresistant Staphylococcus aureus. - Am J Emerg Med, 2012;30(6):1013.e1-4.

7. Hayes, M., D.White, A. Richards. Secondary syphilis presenting as atypical oral ulceration--a case report. - Dent Update, 2008, 35(7):465-7.

8. Husein-Elahmed, H., J.C. Ruiz-Carrascosa. Secondary syphilis presenting as rash and annular hyperkeratotic lesions. - Int J Infect Dis, 2011;15(3):e220.

9. Kashiwada, T., K. Kikuchi, S. Abe et al. Staphylococcal enterotoxin B toxic shock syndrome induced by community-acquired methicillin-resistant Staphylococcus aureus (CAMRSA). - Intern Med, 2012;51(21):3085-8.

10. Khammassi, N., H. Abdelhedi, D. Mohsen, J. Chrifi, M.N. Tougourti, M. Hamza. Disseminated tuberculosis in two immunocompetent patients. Tunis Med, 2009;87(10):717-9.

11. Komiya, K., H. Ariga, N. Nagayama et al. A case of cold abscess of the chest wall due to thoracic drainage for tuberculous pleuritis. - Kekkaku, 2010;85(8):673-7.

12. Mahajan, V.K., N.L. Sharma. Scarlet fever. Indian Pediatr, 2005;42(8):829-30.

13. Marrie, T,J. Osler's nodes and Janeway lesions. Am J Med, 2008;121(2):105-6. 
14. Mota-Burgos, A., A.V. Villa, A. Noguera-Julian, C. Fortuny, M.A. González-Enseñat. Fever and skin lesions in a healthy 6-month-old boy. Diagnosis: Ecthyma gangrenosum. - Pediatr Infect Dis J, 2012;31(7):789-94.

15. Piso, R.J., S. Bassetti. Skin and soft tissue infections. - Praxis (Bern 1994), 2012;101(6):399-405.

16. Silva, N., M.E. Eremeeva, T. Rozental et al. Eschar-associated spotted fever rickettsiosis, Bahia, Brazil. - Emerg Infect Dis, 2011;17(2):275-8.

17. Tully, A.S., K.P. Trayes , J.S. Studdiford. Evaluation of nail abnormalities. - Am Fam Physician, 2012;85(8):779-87.

18. Yokota, S., K. Kawabe, H. Yamada, M. Nunomura. Case of subcutaneous abscess caused by Nocardia farcinica in an aplastic anemia patient. - Nihon Ishinkin Gakkai Zasshi, 2010;51(2):93-7.

19. Zheng, H., C. Ye, M. Segura, M. Gottschalk, J. $\mathrm{Xu}$. Mitogenic effect contributes to increased virulence of Streptococcus suissequence type 7 to cause streptococcal toxic shock-like syndrome. Clin Experiment Immunol, 2008;153(3):385-91. 